ALPHA N² 28 Julio 2009 (87-104)

ISSN 0716-4254

http://alpha.ulagos.cl

\title{
NIETZSCHE Y LA LIBERTAD INDIVIDUAL: RAWLS, CAVELL Y EL DEBATE SOBRE EL VALOR DEL PERFECCIONISMO PARA LA DEMOCRACIA
}

Nietzsche and individual freedom: Rawls, Cavell and the debate on the value of improvement for democracy ${ }^{1}$

Vanessa Lemm*

Resumen

Este artículo rastrea el debate sobre el valor del perfeccionismo en la teoría democrática contemporánea a través de una discusión de las interpretaciones controvertidas de John Rawls y Stanley Cavell de Schopenhauer como educador de Friedrich Nietzsche. El artículo argumenta que las lecturas del perfeccionismo de Cavell y Rawls captan pobremente la noción nietzscheana de libertad individual como responsabilidad. Asimismo, sugiere que esta noción de Nietzsche puede resultar valiosa para la política democrática en virtud, y no a pesar, de constituirse como una alternativa a la versión liberal de la cultura y de la libertad individual.

Palabras clave: Perfeccionismo, Nietzsche, cultura, libertad individual, democracia.

\section{Abstract}

This article traces the debate on the value of improvement in contemporary democratic theory through a discussion of the controversial interpretations of John Rawls and Stanley Cavell of Schopenhauer as Educator by Friedrich Nietzsche. It argues that the readings of perfectionism offered y both Rawls and Cavell poorly capture the Nietzschean notion of individual freedom as responsibility. The latter may turns out to be of value for modern democratic life not in virtud of, but despite the fact that it constitutes an alternative to the liberal conceptions of culture and individual freedom.

Key words: Improvement Nietzsche, culture, individual freedom, democracy.

\section{INTRODUCCIÓN}

En el presente trabajo me propongo rastrear la cuestión del perfeccionismo en la teoría democrática contemporánea. Mi punto de partida es la

${ }^{1}$ Este artículo forma parte de una investigación financiada por Fondecyt Chile: Proyecto N 1085238 "Nietzsche, Biopolitics and the Future of the Human”. 


\section{Vanessa Lemm}

controversia entre John Rawls y Stanley Cavell acerca de las interpretaciones del perfeccionismo en la filosofía de la cultura de Friedrich Nietzsche, específicamente en su obra Schopenhauer como educador. ${ }^{2}$ Esta controversia fue iniciada por una famosa nota al pie de Teoría de la justicia (1971) de John Rawls en la cual cita un fragmento del texto de Nietzsche con el propósito de ilustrar los principios del perfeccionismo político a los que, a posteriori, condena como "intrínsecamente no democráticos y elitistas" (Rawls, 1971:325, nota 51; Cavell, 1990:3-56). Cabe destacar, que la mencionada nota de Rawls, adquirió gran relevancia y suscitó una vasta literatura porque fue ocasión de la célebre defensa del perfeccionismo moral llevada a cabo por Cavell bajo el influjo de las filosofías de la cultura de Nietzsche y de Emerson. Según Cavell, el perfeccionismo moral no sólo es indispensable para dar forma a una crítica interna de la democracia sino que, además, resulta esencial para "la vida de la justicia en una democracia constitucional” (Cavell, 1900:3;56). ${ }^{3}$ En el centro del debate sobre el perfeccionismo se encuentra el problema de hasta qué punto una defensa de valores aristocráticos puede ser

2 Stanley Cavell. Conditions Handsome and Unhandsome: The Constitution of Emersonian Perfectionism. Chicago: University of Chicago Press, 1990 (Cfr. John Rawls. A Theory of Justice). Cambridge, Massachusetts: Harvard University Press, Belknap Press, (1971):325-332. Para otras lecturas de la filosofía de la cultura de Nietzsche como un ejemplo del perfeccionismo, político o moral, véase Daniel, D. Conway. Nietzsche and the Political. Florence, KY: Routledge, 1997; James Conant, "Nietzsche's Perfectionism”, en Richard Schacht (ed.): Nietzsche's Postmoralism. Essays on Nietzsche's Prelude to Philosophy's Future. Cambridge: Cambridge University Press, (2001):181-257, David Owen, “Equality, Democracy, and SelfRespect”: Reflections on Nietzsche’s Agonal Perfectionism, en Journal of Nietzsche-Studies 24 (2002):113-131 and Herman W. Siemens, "Agonal Communities of Taste: Law and Community in Nietzsche's Philosophy of Transvaluation”, en Journal of Nietzsche Studies 24 (2002):83-112. Para un tratamiento anterior de la pregunta si Nietzsche advoca una forma de perfeccionismo, véase Vanessa Lemm, "Is Nietzsche a Perfectionist?: Rawls, Cavell and the Politics of Culture in Schopenhauer as Educator”, en Journal of Nietzsche Studies 34 (2007):527.

${ }^{3}$ Sobre Cavell y la filosofía política, véase Richard Shusterman. "Putnam and Cavell on the Ethics of Democracy”, en Political Theory, Vol. 25, N 2 (Apr., 1997):193-214; David Owen. "Cultural Diversity and the Conversation of Justice: Reading Cavell on Political Voice and the Expression of Consent”, en Political Theory, Vol. 27, No 5 (Oct., 1999):579-596; Andrew Norris. "Political Revisions: Stanley Cavell and Political Philosophy", en Political Theory, Vol. 30, $\mathrm{N}^{\circ} 6$ (Dec., 2002):828-851; Hans von Rautenfeld. “Charitable Interpretations: Emerson, Rawls, and Cavell on the Use of Public Reason”, en Political Theory 32, № 1 (2004):61-84 y The Claim to Community. Essays on Stanley Cavell and Political Philosophy, editado por Andrew Norris. Stanford, California: Stanford University Press, 2006. Para una discusión del valor de la filosofía de Nietzsche para la democracia, véase Bonnie Honig, Political Theory and the Displacement of Politics. Ithaca and London: Cornell University Press, (1993):42-75; Lawrence Hatab. A Nietzschean Defense of Democracy. Chicago: Open Court, 1995 y Wendy Brown, "Nietzsche for Politics", en Alan D. Schrift (ed.), Why Nietzsche Still? Berkeley and Los Angeles: University of California Press (2000):205-223. 
asimilada a una concepción liberal de la justicia. El intercambio entre Cavell y Rawls resulta significativo, no sólo porque echa luz sobre la cuestión recurrente de la interpretación de Nietzsche, sino porque, además, alude directamente a cuestiones de fondo respecto al carácter político de la libertad individual en la sociedad democrática moderna, es decir, hasta qué punto la libertad individual es de naturaleza democrática o aristocrática.

A continuación, propongo que las lecturas del perfeccionismo de Cavell y Rawls captan pobremente la noción nietzscheana de libertad individual como responsabilidad. Asimismo, sostengo que esta noción de Nietzsche puede resultar valiosa para la política democrática en virtud, y no a pesar, de constituirse como una alternativa a la versión liberal de la cultura y de la libertad individual. ${ }^{4}$

\section{PERFECCIONISMO MORAL: IGUALITARIO Y DEMOCRÁTICO (CAVELL) V/S PERFECCIONISMO POLÍTICO: ELITISTA, Y ANTI-LIBERAL (RAWLS)}

En Teoría de la justicia, Rawls caracteriza a una versión extrema de perfeccionismo como una teoría teleológica cuyo principio rector es conducir a "la sociedad hacia un arreglo de instituciones y hacia una definición de deberes y obligaciones de los individuos orientados a maximizar el logro de la excelencia humana en el arte, la ciencia y la cultura” (Rawls, 1971:325). Rawls sostiene que el pensamiento de Nietzsche es un ejemplo de esta

\footnotetext{
${ }^{4}$ Este ensayo se limita a dar un análisis de Schopenhauer como educador. Para un intento de leer la obra entera de Nietzsche como un perfeccionismo, Cfr. Nietzsche and the Political de Conway. La interpretación de Conway sobre Nietzsche como un perfeccionista moral y político es particularmente interesante porque reconcilia las perspectivas en conflicto de Rawls y Cavell sobre el perfeccionismo de Nietzsche. Conway distingue en Nietzsche entre un perfeccionismo político y uno moral y sostiene que mientras el perfeccionismo político caracteriza a los escritos pre-zarathustrianos de Nietzsche, y se manifiesta como una política de revolución y redención que apunta a "incarnar, un complemento expandido de los poderes y perfecciones humanas” a través de una legislación política (Conway, Nietzsche and the Political, 6) el perfeccionismo moral caracteriza a los escritos post-zarathustrianos de Nietzsche y se manifiesta como una forma más moderada y modesta de perfeccionismo que toma la forma de una política de la resistencia. La división que Conway hace del pensamiento político de Nietzsche en un antes y un después de Zarathustra ha sido cuestionado por Hatab en una respuesta a Conway. Cfr. Conway, "The Politics of Decadence”, en The Southern Journal of Philosophy, Vol. XXXVII, 1999:19-33 y Hatab. "Time-sharing in the Bestiary: In Daniel W. Conway's "Politics of Decadence", en The Southern Journal of Philosophy, Vol. XXXVII 1999:35-41. La distinción de Conway también va en contra de la lectura del pensamiento político de Nietzsche propuesta por Keith Ansell-Pearson en An Introduction to Nietzsche as Political Thinker. Cambridge: Cambridge University Press, 1994. Según Ansell-Pearson, el pensamiento político de Nietzsche se vuelve mucho más aristocrático (y no más moderado como sostiene Conway) culminando en el aristocratismo radical de Más allá del bien y el mal (1988) y El Anticristo (1988).
} 
definición de perfeccionismo e ilustra su afirmación citando, en nota al pie, el siguiente fragmento de Schopenhauer como educador

La humanidad debe trabajar continuamente para producir grandes individuos humanos ésta y no otra es la tarea... porque la pregunta es: ¿Cómo puede tu vida, la vida individual, conservar su valor más alto, su sentido más profundo?... Solamente viviendo para el bien de los especímenes más extraordinarios y valiosos (Rawls, 1971:325, nota 51 y Cavell, 1990:49). ${ }^{5}$

Observemos que Rawls rechaza el principio de perfección porque entra en conflicto con el axioma, expresado en el primer principio de justicia, que postula el mismo valor moral para todos los individuos. Según este principio, todos los individuos son merecedores de un esquema de derechos igualitario. ${ }^{6}$ Por el contrario, con el propósito de rehabilitar el valor del perfeccionismo para una teoría de la justicia liberal, Cavell retorna al mismo fragmento citado por Rawls y demuestra que contiene todos los rasgos de un perfeccionismo moral que es intrínsecamente igualitario y democrático.

El argumento de Cavell está construido alrededor de dos problemas centrales. El primero tiene que ver con la concepción errónea que atribuye Rawls sobre el rol de la cultura en Nietzsche. Cavell sostiene que "la vida de la cultura” en Nietzsche no puede tomar la forma de una organización social y política que privilegia a una minoría cultivada al costo de mantener sin cultivar a una mayoría — como sugiere Rawls - porque, a su juicio, la "vida de la cultura" pertenece al individuo y permanece al cuidado exclusivo del individuo para su propio bien (1990:51). Para Cavell, la cultura no es política

\footnotetext{
${ }^{5}$ La cita de Nietzsche en Rawls y Cavell es inexacta. Me refiero aquí a lo que citan Rawls y Cavell con el propósito de examinar sus respectivas lecturas. Vuelvo a esta cita en un momento.

${ }^{6}$ El primer principio de justicia en Rawls dice: “cada persona ha de tener un derecho igual a la más amplia y equitativa disposición de libertades básicas, compatible con una similar disposición de libertades de los otros”. A Theory of Justice (Revised Edition). Cambridge: Harvard University Press (1999:53). Simone Chambers comenta la presuposición de este primer principio en el trabajo de Rawls: "Podemos discernir tres esferas entrelazadas de igualdad en los escritos de Rawls: la igualdad fundamental, la igualdad política y la igualdad social y económica. La igualdad fundamental parte de la reivindicación acerca del estatus moral de los individuos, a saber, que todas las personas tiene una igual dignidad... Tres puntos se sostienen acerca de la igualdad fundamental: es notoriamente difícil de demostrar, no es evidente por sí mismo lo que sigue de dicha reivindicación; y es lo más cercano a lo que una democracia liberal tiene como axioma". Simone Chambers. "The Politics of Equality: Rawls on the Barricades”, en Perspectives on Politics vol. 4, $\mathrm{N}^{\circ} 1$ (2006:82). Estos tres puntos reflejan muy bien las razones que estimulan el debate de Cavell-Rawls sobre el perfeccionismo de Nietzsche.
} 
sino que se trata de una cuestión privada: es la vida "vivida para el bien de quien la vive” (1990:51). De tener esta vida un vicio característico, éste sería una cierta "reducción de la participación democrática" (1990:51). Pero esta participación limitada no transforma a esta vida, sin más, en una vida no liberal o anti-democrática. Por el contrario, la vida de la cultura es un ejemplo de uno de los principios de la propia teoría de la justicia liberal de Rawls: el principio de que ser libre implica vivir de acuerdo a una determinada concepción del bien como mejor le parezca a cada uno. Más aún, según Cavell, la vida de la cultura en Nietzsche (y en Emerson) expresa el valor intrínseco del individuo, su capacidad para "consagrar lo que ha sido logrado hasta el momento al yo que aún ha de lograrlo" y, por lo tanto, es una forma de vida que dista de ser inmoral (1990:51). Por último, Cavell propone que dada la capacidad moral del individuo para autoperfeccionarse - a la que identifica con el genio individual - y puesto que esta capacidad se halla siempre ya "distribuida universalmente", se deduce que la cultura es intrínsecamente igualitaria y no elitista.

El segundo problema que identifica Cavell en la interpretación que hace Rawls del perfeccionismo de Nietzsche tiene que ver con la forma como este autor se apropia del término "espécimen (Exemplare)”. Según Cavell, Rawls considera que "el espécimen más valioso" (en la cita de Nietzsche ya mencionada al principio de esta sección) se refiere a aquellos miembros de una minoría privilegiada que viven a expensas de la mayoría y que requieren que ésta se sacrifique por el bien de la minoría, incrementando así el valor de sus vidas. En contra de esta interpretación, Cavell afirma que "espécimen” no alude a una clase o grupo, sino que refiere a "mi propio yo" entendido como otro individuo. Cavell halla sustento para esta conclusión en la traducción, supuestamente errónea, del alemán "Exemplare” al inglés "specimen”. Una vez corregido el error Exemplare pasa a ser "ejemplar" y no "espécimen". Resulta claro, entonces, para Cavell que en Nietzsche la relación con un "ejemplar" no implica el imperativo político de que una clase deba sacrificarse en beneficio de otra clase "superior". Por el contrario, para Cavell la relación con un ejemplar refleja la relación del individuo con "su propio yo superior"; con el yo en el que aspira uno a convertirse (1990:50-51). Según Cavell, la relación que el yo establece con su propio yo superior refleja la perspectiva del yo sobre sí mismo y no su visión de los otros. Así, para Nietzsche la cultura debe ocuparse de la aculturación del yo individual y no de la organización política y social, como sugiere Rawls.

Para Nietzsche, lo que sitúa al individuo en el círculo de la cultura es el apego a un gran ser humano. Sin embargo, para Cavell, el amor por "un gran hombre” no constituye una relación con otra persona como fin en sí mismo, sino que se trata de un medio para la perfección del propio yo (1990:53). "El 
apego de nuestro corazón” devuelve un reflejo negativo en el individuo, revelando las limitaciones del propio yo y estimulando su deseo de aspirar a algo superior. En la perspectiva de Cavell, en el yo se originan tanto este verse reflejado negativamente como la aspiración a un yo superior. Puesto que, desde esta perspectiva, el "más allá del yo” es sólo siempre un más allá al interior del yo, se deduce que el yo no se halla apegado a un gran ser humano sino a su propio yo (superior). Cavell subraya que "lo superior", el deseo por el cual es creado en la propia insatisfacción, se halla inscripto como "un yo superior todavía oculto" y agrega que este último no refiere a "alguien más" sino que apunta a "mi propio y perturbadoramente inalcanzado" yo (1990:51). El punto esencial del argumento de Cavell reside en que si la relación entre el yo y el ejemplar es una relación entre el yo y su yo (superior), entonces, se trata de una relación entre iguales y prueba que el perfeccionismo moral no es elitista sino igualitario. Por lo tanto, Cavell debe rechazar una interpretación de Nietzsche que asimile la relación entre ejemplar e individuo a la relación entre maestro y alumno. En una concepción de la cultura igualitaria, la relación con el ejemplar no puede ser discipular porque ésta instituye una desigualdad que no puede ser reconciliada con una teoría de la justicia liberal.

Para comprender cabalmente la intención de Cavell en su laborioso encuentro con Teoría de la justicia es importante destacar que el interés que despierta en Cavell el rechazo del perfeccionismo en Rawls no indica, al menos no directamente, que el primero se halle interesado en el pensamiento de Nietzsche. El interés de Cavell reside, en cambio, en la filosofía norteamericana y su relación con Emerson. La "defensa” que hace Cavell de Nietzsche es, en realidad, una defensa del perfeccionismo moral emersoniano, pero constituye, al mismo tiempo, una defensa que pretende salvar a Teoría de la justicia de sí misma (1990:4). Cavell afirma que el presupuesto de Rawls de un valor moral equivalente para todos los individuos sólo generará justicia en la sociedad si es reconocido y realizado por los miembros individuales de esa sociedad, y no solamente por su estructura básica (1990:56-57). Cavell le sugiere a Rawls que la brecha entre justicia en teoría (esto es, justicia como se aplica a instituciones) y justicia en la práctica (esto es, justicia en tanto aplicada a individuos) puede ser mediada a través de la práctica individual del perfeccionismo moral emersoniano. Por lo tanto, este último podría convertirse en "parte del entrenamiento para la democracia" (1990:56).

\section{EL PROBLEMA DE LA CULTURA}

Luego de presentar sintéticamente las visiones enfrentadas de Rawls y Cavell resulta útil volver al comienzo, es decir, a la cita de Nietzsche que 
originó la disputa en cuestión. Efectivamente, el fragmento de Schopenhauer como educador que se encuentra en Rawls y en la lectura que de éste hace Cavell no es exacta. En este fragmento, Nietzsche comienza invitando al lector a reflexionar con él sobre una proposición suya a la que incluye como cita, pero que ni Rawls ni Cavell reconocen como tal. La cita en cuestión es la siguiente: "La humanidad debe trabajar continuamente en la producción de grandes individuos humanos ésta y no otra es la tarea” (Nietzsche, 2001:75). Resulta difícil determinar a esta altura del texto hasta qué punto esta proposición pertenece realmente a Nietzsche o si se trata de una mera cita destinada a abrir la discusión. Según Nietzsche, la cita refleja el intento de aplicar a la sociedad y sus metas lo que puede aprenderse de la observación de cualquier especie (Art) del mundo animal o vegetal: que su única preocupación es el ejemplar individual superior (einzelne höhere Exemplare), el más extraordinario, el más poderoso, el más complejo, el más provechoso.

Nietzsche reconoce que la aplicación de esta fórmula a la sociedad resulta difícil porque genera una "fuerte resistencia". Las razones para esta resistencia son obvias: "Que un ser humano deba existir sólo para el propósito de otro ser humano parece una exigencia absurda” (2001:76). En cambio, "un ser humano" debería existir para todos los demás o bien, como apunta Nietzsche, al menos para tantos como sea posible. Pero Nietzsche se pregunta ¿Es el segundo requerimiento menos absurdo que el primero? “ $i$ Como si fuese menos absurdo dejar decidir a los números cuando se discute sobre valor y sentido!” (2001:76). Frente a este dilema, Nietzsche reformula la proposición inicial en sus propias términos: "Porque la pregunta es la siguiente: ¿Cómo puede tu vida, la vida del individuo (des Einzelnen Leben), conservar (erhält) el valor más alto y el sentido más profundo?” (2001:76). Sugiero que es ésta cuestión, y no la proposición citada al comienzo del fragmento, la que ilumina la concepción de Nietzsche sobre la cultura. Ambas proposiciones se entremezclan tanto en la cita de Rawls y, en su comentario, como en la glosa que hace Cavell. ${ }^{7}$

No podré discutir, aquí, todos los significados de la pregunta nietzscheana “¿Cómo puede tu vida, la vida del individuo, conservar el valor más alto y el sentido más profundo?”. ${ }^{8}$ Me limitaré, entonces, a lo que estimo es uno de sus aspectos más importantes, es decir, la relación entre "el valor más alto y el sentido más profundo" del individuo y su responsabilidad. En la sección inicial de Schopenhauer como educador, Nietzsche formula el

\footnotetext{
${ }^{7}$ Acerca de esta confusión en Rawls y Cavell, Cfr. Conway. Nietzsche and the Political, 53.

${ }^{8}$ Para una discusión más extensa de esta pregunta (Cfr. Vanessa Lemm. "Is Nietzsche a Perfectionist? Rawls, Cavell and the Politics of Culture in Nietzsche's Schopenhauer as Educator”) en Journal of Nietzsche Studies 34 (2007:5-27).
} 
problema de la libertad en relación con el de la responsabilidad en términos de un desafío existencial que nos incumbe a todos

pero aun cuando el futuro no nos permitiera esperar nada, nuestra peregrina existencia precisamente en este ahora nos incita con la mayor fuerza a vivir de acuerdo con nuestra propia medida y nuestra propia ley. Lo inexplicable que resulta que vivamos precisamente hoy habiendo, no obstante, tenido todo el tiempo infinito para venir a ser, que no poseemos más que un apretado hoy y que tengamos que demostrar en él por qué y con qué fin hemos venido a ser hoy y no en otro momento. Tenemos que asumir la responsabilidad sobre nuestra existencia antes nosotros mismos; por ello queremos ser también los verdaderos pilotos de esta existencia sin permitir que se asemeje a un azar inconsciente (2001:27. Las modificaciones de la traducción son mías)

Es digno de destacar que Nietzsche está interesado en un tipo de responsabilidad que no es de naturaleza moral ni política. En realidad, Nietzsche concibe la responsabilidad como algo que es cultivado a-un-lado, y en contra de, las formas institucionalizadas de "cultura oficial”. El carácter anti-institucional de la cultura (y de la responsabilidad) parece favorecer la interpretación de Cavell de la cultura en Nietzsche, según la cual, la cultura no puede ser maximizada a través de la organización política y social, como sugiere Rawls, porque se constituye a través de una crítica a esta organización.

Sin embargo, el carácter anti-institucional de la cultura (y de la responsabilidad) en Schopenhauer como educador entra en conflicto con el argumento de Cavell respecto a que la cultura es "una vida vivida para el bien de quien la vive”, y que este bien refleja una capacidad moral para el autoperfeccionamiento que se halla distribuida de manera igualitaria. Más bien, la cultura representa el cultivo de una práctica de la responsabilidad inseparable de una noción de libertad individual que es intrínsecamente política y que excede al individualismo de Cavell basado en la idea de una "absoluta responsabilidad del yo para sí mismo” (1990:XXVII). Además, la noción de libertad individual presupuesta en la visión de Nietzsche sobre la responsabilidad es intrínsecamente plural y, por ende, incompatible con la idea de una perfección moral que se halla circunscripta a la relación del yo consigo mismo. Por último, la noción de libertad individual en tanto responsabilidad es intrínsecamente aristocrática y, por ende, no puede ser asimilada a 
la idea liberal de libertades individuales que deben ser distribuidas de manera igualitaria. $^{9}$

Para Nietzsche, la responsabilidad es un valor aristocrático que entra en conflicto con la idea liberal de igualdad de derechos porque la responsabilidad constituye un señalamiento del individuo que adopta en cada persona una forma única e irreductible. No puede ser distribuida por igual de la manera que sugiere Cavell porque la responsabilidad es intrínsecamente contingente, es decir, se halla radicalmente situada. A diferencia de la capacidad de autoperfeccionamiento, la responsabilidad no define el valor moral intrínseco del individuo. Si para Rawls, el hecho de que el valor de un individuo es igual al de cualquier otro representa un axioma, si para Cavell, el valor de un individuo reside en el resultado de su progreso hacia la auto-perfección y, por ello, es diferente al valor de otros individuos. Mientras que Rawls y Cavell intentan determinar el valor del individuo, Nietzsche llega a la conclusión de que se trata de una pregunta abierta que no puede responderse de una vez y para siempre. Por el contrario, esta pregunta se halla reconfigu-rada constantemente en ocasión de la confrontación del individuo con el desafío de la responsabilidad. Nietzsche encuentra que no existe una sola manera de obtener el valor más alto y el sentido más profundo, sino que existe un número infinito de formas igualmente valiosas cuya singularidad es irreductible.

En la pregunta “¿Cómo puede conservar (erhält) la vida individual el valor más alto y el sentido más profundo?” (2001:76) Nietzsche usa el verbo "erhalten", que se traduce literalmente como "recibir". En primera instancia, "recibir" indica que el valor y el sentido llegan al individuo y, en segundo lugar, que este movimiento hacia el individuo es un movimiento de retorno. Por lo tanto, valor y sentido no son algo que el individuo lucha por conseguir, como sucede en la visión de Cavell de la cultura como "la consagración de aquello que ha sido logrado hasta el momento al yo que aún ha de lograrlo" (1990:51). Más aún, en contraste con la concepción de Cavell, que entiende el auto-perfeccionamiento como la lucha moral del individuo por alcanzar un yo más elevado al interior del propio yo, valor y sentido no se originan en el individuo ni pertenecen a él. Más bien, el retorno del valor y el sentido hacia el individuo depende de una apertura existencial del individuo hacia el otro. Para Nietzsche, responsabilidad es un nombre de esta apertura, de este exponerse del yo hacia el otro en tanto otro. Cada responsabilidad individual refleja la forma única como el individuo responde a una llamada dirigida al individuo. Es la apertura individual al llamado de la responsabilidad lo que

\footnotetext{
${ }^{9}$ Para una discusión de las diferencias entre el pensamiento político de Nietzsche y el liberalismo, Cfr. Ansell-Pearson. An Introduction to Nietzsche as Political Thinker, 9-13.
} 
confiere a la vida del individuo el valor más elevado y el sentido más profundo.

En mi interpretación, en Nietzsche el término "lucha por la cultura" es otro nombre posible para esta apertura del individuo a la otredad. En palabras de Nietzsche

[L]a cultura requiere del individuo no sólo una experiencia interior, no sólo una evaluación del mundo exterior que fluye a su alrededor, sino que finalmente y sobre todo (hauptsächlich) requiere un acto, es decir, una lucha (Kampf) en nombre de la cultura y una hostilidad hacia esas influencias, hábitos, leyes e instituciones en los que no puede reconocer este objetivo: la generación del genio (Erzeugung des Genius) (2001: 78).

Es a través de una lucha contra los adversarios de la cultura que Nietzsche espera pueda favorecerse el objetivo de ésta, es decir, el hacer surgir al genio. El surgimiento del genio no puede ser atraído mediante el perfeccionismo político (Rawls), porque la cultura es esencialmente antiinstitucional, ni por medio de un perfeccionismo moral (Cavell), porque la cultura es esencialmente una contienda pública que presupone no sólo una pluralidad de agentes, sino también las relaciones en favor y en contra en que estos agentes se ven implicados.

\section{DE GENIOS, EJEMPLARES Y ESPECÍMENES}

Con respecto al segundo problema que identifica Cavell en la lectura de Nietzsche que hace Rawls —el problema de la traducción de "ejemplar" como "specimen"- estoy de acuerdo con Cavell en que el genio en Nietzsche se perfila como un término de "incomparable fecundidad (produktive Einzigkeit)” que se halla igualmente distribuido (Nietzsche, 1988:350;337;8).

De este modo, al menos en lo que concierne al genio, la cultura es democrática e igualitaria. Sin embargo, sostengo que Nietzsche también utiliza el término genio para referirse a algo que se encuentra más allá del yo individual, que viene hacia el yo (como la responsabilidad) y sin el cual el carácter único del yo no podría devenir fecundo. ${ }^{10}$ Mientras que el primer uso

\footnotetext{
${ }^{10}$ Agamben retrae esta doble naturaleza del genio individual a su origen en el dios romano Genius. Agamben argumenta que el genio define nuestra vida en la medida que no nos pertenece. Genio revela la presencia de algo que, aunque no pertenece al individuo, lo diferencia de manera esencial. Giorgio Agamben. Profanierungen. Frankfurt: Suhrkamp (2005:7-17). Para otra interpretación del genio como aquello que no nos pertenece ( $C f r$. Jean-Luc Nancy. "Praesens" en The Gift. Generous Offerings Threatening Hospitality, curado por Gianfranco Maraniello, Sergio Risaliti, Antonio Somaini. Milano: Edizioni Charta (2001:191-199). Vale la
} 
de genio denota el aspecto democrático e igualitario de la cultura en Nietzsche, el segundo uso denota un aspecto aristocrático y no igualitario.

Sugiero que en lugar de interpretar la política de la cultura en Nietzsche o bien como elitista (Rawls) o bien como democrática (Cavell), propongo que debería ser leída, al mismo tiempo, como democrática y aristocrática. ${ }^{11}$ En una época que encuentra lo mismo siempre y en todo lugar, Nietzsche defiende el valor en todos los individuos de aquello que es desigual y que los distingue de los demás. ${ }^{12}$ Mientras que "todos los individuos" denota el aspecto democrático en su concepción de la cultura, "distinción” denota su perfil aristocrático. El rechazo de lo "mismo" en Nietzsche expresa, en cambio, su temor hacia aquello que Adorno y Horkheimer, y que luego también Levinas, identificaron como lo totalitario. ${ }^{13}$ La llamada a "vivir para la generación del genio” (2001:78) articula, entonces, la necesidad de cultivar

pena también notar que el término "Erzeugung” en la frase de Nietzsche "la generación del genio" se refiere a un proceso vital que requiere recibir al otro, en contraste a, por ejemplo, un proceso (manual o mecánico) de producción que procede en ausencia de otros.

${ }^{11}$ Para otros intentos de pensar conjuntamente los aspectos aristocráticos y democráticos de la filosofía de Nietzsche véase Quentin P. Taylor. The Republic of Genius. A Reconstruction of Nietzsche's Early Thought. Rochester: Rochester University Press (1997:48-49;90) Lawrence Hatab. A Nietzschean Defense of Democracy: An Experiment in Postmodern Politics. Chicago: Open Court, 1995 y "Prospects for a Democratic Agon: Why We Can Still Be Nietzscheans", en Journal of Nietzsche-Studies 24 (2002:132-147) Wendy Brown. "Nietzsche for Politics”, en Alan D. Schrift (ed.), Why Nietzsche Still?. Berkeley and Los Angeles: University of California Press (2000:205-223) y Christa D. Acampora. "Demos Agonistes Redux, Reflections on the Streit of Political Agonism”, en Nietzsche-Studien 32 (2003:374-390). Hatab distingue entre “el encuentro aristocrático-democrático en la esfera cultural" y "el encuentro aristocráticodemocrático en la esfera política” (2002:132-147). Si el primero tiene que ver con "cuestiones de creatividad y normalidad, excelencia y mediocridad”, el segundo tiene que ver con la "formación de instituciones, prácticas políticas, la justificación de la coacción y los límites de la soberanía". Hatab mantiene que "el aristocratismo de Nietzsche es defendible respecto del primer tipo de encuentro, pero no respecto al segundo tipo de encuentro" (2002:141). Hatab se pregunta acerca de una posible coexistencia entre una elite cultural Nietzscheana y una política democrática e igualitaria (2002:141). Yo concuerdo con Hatab, pero me gustaría agregar que la coexistencia entre un aristocratismo cultural y una política igualitaria y democrática se puede concebir como una relación agonista que abre la cultura a la democracia (democratiza la cultura) y que abre la democracia a la cultura (cultiva nuevas formas de vida democrática por venir).

12 La sensibilidad de Nietzsche por su tiempo es, en este sentido, comparable a la de Tocqueville. Para un ejemplo clásico de la importancia de los elementos aristocráticos entre la sociedad democrática que trabaja en favor de la preservación de la libertad individual. Cfr. Alexis de Tocqueville. Democracy in America and Two Essays on America. London: Penguin Books, 2003, Vol. 1, segunda parte, capítulo 8.

${ }^{13}$ Max Horkheimer and Theodor W. Adorno. Dialectic of Enlightenment New York: The Continuum Publishing Company (2002:12); Emmanuel Levinas. Totalité et infini. La Haye: Nijhoff, 1961. 


\section{Vanessa Lemm}

una sensibilidad aristocrática que valora la diferencia y la distinción en cada individuo en contra de una sensibilidad (y una política) democrática que sólo encuentra mismidad e igualdad. Una sociedad democrática es incapaz de atribuir el valor más alto y el sentido más profundo a la fecundidad única e incomparable (responsabilidad) de cada individuo. ${ }^{14}$

Lo que distingue al valor de los “ejemplares superiores” en Nietzsche es que su relación con el otro confiere a la vida de cada individuo el valor más alto y el sentido más profundo. Nietzsche toma esta perspectiva de su observación del mundo animal y vegetal donde todo pugna hacia la generación (Erzeugung) de sus ejemplares más extraordinarios, porque éstos elevan el valor de la vida en general (animal y vegetal). ${ }^{15}$ Esta elevación del valor, sin embargo, no se logra por medio de una mayor perfección (de las especies o de la naturaleza) como sugieren la interpretaciones perfeccionistas, sino por medio de un aumento diversificador y pluralizador de la vida. Los ejemplares superiores son importantes para todos los demás porque "agregan a la naturaleza nueva naturaleza viviente (die Natur durch neue lebendige Natur vermehren)" (1988, vol.1:383). En este sentido, lo que confiere el valor más alto y el sentido más profundo a la vida no es el incremento en la perfección moral individual, sino la creciente pluralización y diversificación de la vida. La pluralización de la vida alcanzada por los ejemplares más elevados resulta de tanta importancia para Nietzsche no sólo porque refleja una relación libre con el otro sino, también, porque posee un efecto liberador

\footnotetext{
${ }^{14}$ Mientras que Cavell identifica el esfuerzo por situarse por encima de la norma como un rasgo típico del perfeccionismo, yo lo entiendo como un rasgo característico de la crítica decimonónica a la nivelación y la normalización propias de la sociedad de masas que se halla tanto en la tradición liberal (Emerson, J. S. Mill), como entre sus críticos (Kierkegaard, Dostoievski, Nietzsche).

${ }^{15}$ La interpretación de Cavell del uso del término "Exemplare" en Nietzsche resulta poco convincente debido a la referencia recurrente de Nietzsche al mundo animal y vegetal. Cuando Nietzsche responde la pregunta referida al valor y sentido de la vida individual parece estar aplicando — nuevamente - a la sociedad y sus propósitos "algo que puede ser aprendido a partir de la observación de cualquier especie (Art) del mundo animal y vegetal” (2001:75). La continuidad entre la vida de las plantas, los animales y el animal humano puede explicar por qué, para Nietzsche, la respuesta debe ser "con seguridad”: "sólo mientras vivas al servicio de los ejemplares más valiosos y extraordinarios” (2001:75). Sin embargo, concuerdo con Cavell en que el término inglés "specimen" traduce pobremente el alemán "Exemplar" y que sus connotaciones biologicistas no están de acuerdo con la concepción de Nietzsche de la vida como algo que no puede ser dividido en especies (1988 vol. 12:383ss, nota 9[91]). Kaufmann también sostiene que la referencia de Nietzsche a la naturaleza no es biologicista, pero por razones diferentes. Argumenta que Nietzsche aprueba la cultura como una naturaleza (physis) nueva y mejorada, y que, por lo tanto, encuentra en esta última no la continuidad entre humano, animal y vegetal, sino la "obvia" diferencia entre ellos (Kaufmann, Nietzsche: Philosopher, Psychologist, Antichrist 156). Sobre Nietzsche y la biología, Cfr. Barbara Stiegler. Nietzsche et la biologie. Paris: P.U.F., 2001.
} 
en el otro. La libertad que vincula al yo con el ejemplar más elevado, y no con el ejemplar moralmente superior, es la que aumenta el valor y profundiza el sentido de la vida del individuo.

Desde mi perspectiva, el término "Exemplar", tal y como es usado por Nietzsche, no constituye una referencia a la noción kantiana de ejemplaridad, como sugiere implícitamente Cavell (1990:51) o Conant (2001:192ss), lo cual no implica que no existan referencias respecto a la idea de una vida ejemplar en Nietzsche. Por el contrario, Nietzsche se apropia de la idea de vida ejemplar cuando afirma que "El filósofo me beneficia sólo si puede servir de ejemplo (Beispiel)” (1988, vol.1:350). Sin embargo, no es Kant el que inspira la concepción nietzscheana de una vida ejemplar sino los griegos, quienes enseñaban

mediante gestos, con el rostro, con la actitud, con los ropajes, alimentos y costumbres más que con la palabra o la escritura. ¡Cuánto no nos faltará en Alemania de esta valerosa visibilidad de una vida filosófica! (2001:40).

No sólo la noción kantiana de “ejemplaridad” no actúa como fuente de inspiración para la idea nietzscheana sino que, además, Nietzsche cita la vida de Kant como contra-ejemplo de la valiente visibilidad de la vida ejemplar de Schopenhauer como filósofo.

Sugiero que, si algo de Kant logró inspirar el proyecto de Schopenhauer como educador es el famoso ensayo de Kant ¿Qué es la Ilustración? Es digno de destacar que Schopenhauer como educador comienza en forma muy similar al ensayo de “¿Qué es la Ilustración?”. Al igual que Kant, Nietzsche identifica un problema de su época (la falta de ilustración en el caso de Kant y la falta de genio en el caso de Nietzsche) simultáneamente como un problema individual y socio-político. Más aún, Nietzsche —al igual que Kant- define este problema en términos de pereza y falta de coraje. Nietzsche, de manera similar a Kant, alienta a sus lectores a "atreverse a conocer" para superar la pereza y la falta de coraje a través de la búsqueda del conocimiento de sí mismo. Además, en paralelo a Kant, Nietzsche hace responsable a la sociedad por la falta de ilustración (Kant) y por la escasa estima por el genio (Nietzsche). La sociedad "el tutelaje de los guardianes", afirma Kant, ha transformado a los seres humanos en "animales domésticos", en "animales de rebaño" como notará Nietzsche luego, quien afirma que "la opinión pública" los ha transformado en "pseudo humanos" (1988, vol. 1:246). A partir de este momento, las perspectivas de Kant y Nietzsche divergen: Kant es particularmente crítico de toda institución, como por ejemplo la Iglesia Cristiana, basada en el principio de autoridad, y argumenta que la ilustración, 
es decir, la práctica del pensamiento libre, sólo puede ser favorecida a través del cultivo de un espacio público donde académicos y todo aquél que lee y escribe estén invitados a ejercer públicamente su capacidad crítica ante los demás. Casi un siglo después de Kant, Nietzsche ve este espacio público como habiéndose degenerado en un espacio dominado por la opinión pública y prescribe abstenerse de participar en la formación de dicha opinión. No obstante, esta retirada de la esfera pública en Nietzsche no es definitiva, porque lo que más se necesita en la era de la opinión pública es aquello que Nietzsche denomina pensamiento intempestivo. Es, en este punto, donde confluyen nuevamente las posiciones de Kant y Nietzsche, porque ¿Qué otra cosa es el pensamiento intempestivo sino la práctica libre del pensamiento crítico frente a, y en contra de, los demás?

\section{SOBRE LA NATURALEZA POLÍTICA DE LA LIBERTAD INDIVIDUAL}

Para concluir, me gustaría volver a la importancia de la polémica entre las interpretaciones de Nietzsche que presentan Rawls y Cavell relativa a la cuestión fundamental del carácter político de la libertad individual. Específicamente, me gustaría retornar a la cuestión de hasta qué punto la libertad individual es de naturaleza democrática o aristocrática. Lo que diferencia a las posiciones de Rawls y de Cavell de la posición de Nietzsche es que mientras los dos primeros abordan el carácter político de la libertad individual desde el presupuesto de dos tipos de individualismo —uno más igualitario, el otro más perfeccionista- Nietzsche, en cambio, lo aborda a través de una concepción política de la cultura concebida como un asunto público que presupone una pluralidad de agentes implicados en relaciones agonísticas.

En la perspectiva de Rawls, el igualitarismo de un sistema político con los mismos derechos y oportunidades para todos ofrece las condiciones más favorables para la realización de la libertad individual. Cavell coincide con Rawls en este punto, pero sugiere que este esquema de derechos necesita ser complementado con la idea de perfección moral. A pesar de la aparente diferencia entre sus visiones de la libertad individual, para ambos autores aquello que es "político" acerca de la libertad del individuo se asienta en un rasgo del yo: en Rawls toma la forma de la igualdad moral de todos los individuos, mientras que en Cavell se presenta como la diferenciación moral del yo de los otros "yoes", incluyendo la alteridad de su propio yo.

Resulta interesante el hecho de que los individualismos que definen tanto a la posición de Rawls como a la de Cavell también impregnan sus lecturas de Nietzsche. En la lectura de Cavell, la perfección del yo es un emprendimiento privado y moral, en la versión de Rawls se trata de un 
objetivo de la organización política y social, pero ambos encuentran en Nietzsche una concepción liberal de la libertad que toma la forma de un individualismo circunscripto a la autoperfección del individuo. Pero si "perfeccionismo" implica un discurso en el que el valor más elevado es el auto-perfeccionamiento del yo, entonces, Nietzsche no es un perfeccionista. En cambio, la libertad individual, de acuerdo a Nietzsche, tiene lugar a través de la práctica de una política agonística de la responsabilidad y, por ello, excede a la realización de la justicia por medio del auto-perfeccionamiento moral individual (Cavell) sea a la realización mediante la constitución de una "estructura básica” justa de la sociedad (Rawls). Para Nietzsche, la realización de la justicia depende de la participación antagonista de los individuos de una sociedad en favor y en contra de la "estructura básica” de la sociedad.

Por nuestra parte, en contraposición a Rawls y Cavell, aquello que es "político" en la libertad individual de Nietzsche no proviene del yo sino de su lucha por la cultura. Desde esta perspectiva, la cultura no es una posesión ni pública ni privada, es decir, algo que define al estilo de vida de un individuo o de una sociedad. En cambio, la cultura es una lucha pública que genera no sólo la libertad del individuo, sino también la libertad de la sociedad. En esta interpretación, la libertad individual llega al individuo en virtud de su capacidad crítica frente a la autoridad institucionalizada de las normas sociales, morales y políticas de su tiempo. La libertad, al devenir a través de la acción, distingue a un individuo de los otros y, por ende, es una libertad intrínsecamente aristocrática.

Por lo demás, lo que distingue al carácter político de la libertad individual en Nietzsche es la imposibilidad intrínseca de separarla de la responsabilidad, por lo que cuanto mayor es la libertad individual la responsabilidad resulta, también, mayor.

La libertad individual es inseparable de la libertad de la sociedad al punto que no sólo surge de, sino que también concierne a, la relación (libre y responsable) del yo con el otro. Mientras que la libertad y la responsabilidad en los individualismos de Rawls y Cavell pueden impactar indirectamente en la vida política de la sociedad, para Nietzsche la libertad y la responsabilidad generadas por la cultura influyen directamente en las pautas morales, sociales y políticas de la sociedad. Por ende, nunca se trata de una cuestión privada, sino que es siempre, ya, una cuestión pública y política.

Por ultimo, la lucha de la cultura entendida como generación de relaciones libres refleja una preocupación por el futuro, por los "beneficios de un tiempo venidero" (1988, vol. 1:247). El propósito de la cultura es el de superar una forma de vida social y política dada y generar variaciones y alteraciones que ennoblezcan las formas de vida más libres por venir. Es en 
esta función que la política de la cultura agrega valor y sentido a la vida del individuo que vive en democracia.

Universidad Diego Portales* Instituto de Humanidades

Res.: Avda. Alejandro Fleming 11020, casa H4 Las Condes, Santiago (Chile) vanessa.lemm@udp.cl

\section{BIBLIOGRAFÍA}

ACAMPORA, Christa D. "Demos Agonistes Redux, Reflections on the Streit of Political Agonism”, en Nietzsche-Studien 32 (2003):374-390.

AGAMBEN, Giorgio. Profanierungen. Frankfurt: Suhrkamp, 2005.

ANSELL-PEARSON, Keith. An Introduction to Nietzsche as Political Thinker. Cambridge: Cambridge University Press, 1994.

BROWN, Wendy. "Nietzsche for Politics", en Alan D. Schrift (ed.). Why Nietzsche Still. Berkeley and Los Angeles: University of California Press, (2000):205-223.

CAVELL, Stanley. Conditions Handsome and Unhandsome: The Constitution of Emersonian Perfectionism. Chicago: University of Chicago Press, 1990.

CHAMBERS, Simone. "The Politics of Equality: Rawls on the Barricades", en Perspectives on Politics Vol. 4, $N^{\circ} 1$ (2006).

CONANT, James. "Nietzsche's Perfectionism”, en Richard Schacht (ed.). Nietzsche's Postmoralism. Essays on Nietzsche's Prelude to Philosophy's Future. Cambridge: Cambridge University Press. (2001):181-257.

CONWAY, Daniel D. "The Politics of Decadence”, en The Southern Journal of Philosophy, Vol. XXXVII (1999):19-33.

------ Nietzsche and the Political. Florence, KY: Routledge, 1997.

HATAB, Lawrence. "Prospects for a Democratic Agon: Why We Can Still Be Nietzscheans”, en Journal of Nietzsche-Studies 24 (2002):132-147.

------ "Time-sharing in the Bestiary: In Daniel W. Conway's "Politics of Decadence", en The Southern Journal of Philosophy, Vol. XXXVII, (1999):35-41.

------ A Nietzschean Defense of Democracy: An Experiment in Postmodern Politics. Chicago: Open Court, 1995.

HONIG, Bonnie. Political Theory and the Displacement of Politics. Ithaca and London: Cornell University Press, 1993. 
Nietzsche y la libertad individual

HORKHEIMER, Max; ADORNO, Theodor W. Dialectic of Enlightenment. New York: The Continuum Publishing Company, 2002.

KAUFMANN, Walter. Nietzsche: Philosopher, Psychologist, Antichrist. Princeton: Princeton University Press, 1974.

LEMM, Vanessa. "Nietzsches Vision einer "neuen Aristokratie”, en Deutsche Zeitschrift für Philosophie 56 (2008):365-383.

"Is Nietzsche a Perfectionist?: Rawls, Cavell and the Politics of Culture in Schopenhauer as Educator", en Journal of Nietzsche Studies 34 (2007):5-27.

LEVINAS, Emmanuel. Totalité et infini. La Haye: Nijhoff, 1961.

NANCY, Jean-Luc. "Praesens”, en The Gift. Generous Offerings Threatening Hospitality. Curado por Gianfranco Maraniello, Sergio Risaliti, Antonio Somaini. Milano: Edizioni Charta. (2001:191-199).

NIETZSCHE, Friedrich. Schopenhauer como educador. Traducción Jacob Muñoz. Madrid: Bilbioteca nueva, 2001.

------- Kritische Studienausgabe in 15 Bänden. Ed. Giorgio Colli and Mazzino Montinari. Berlin: De Gruyter, 1988.

NORRIS, Andrew. Ed. The Claim to Community, Essays on Stanley Cavell and Political Philosophy. Stanford, California: Stanford University Press, 2006.

------- "Political Revisions: Stanley Cavell and Political Philosophy”, en Political Theory, Vol. 30, ํㅡ 6 (Dec., 2002):828-851.

OWEN, David. "Equality, Democracy, and Self-Respect': Reflections on Nietzsche’s Agonal Perfectionism”, en Journal of Nietzsche-Studies 24 (2002):113-131.

------- "Cultural Diversity and the Conversation of Justice: Reading Cavell on Political Voice and the Expression of Consent", en Political Theory, Vol. 27, N 5 (1999):579-596.

RAUTENFELD, Hans Von. "Charitable Interpretations: Emerson, Rawls, and Cavell on the Use of Public Reason", en Political Theory 32, $\mathrm{N}^{\circ} .1$ (2004):61-84 .

RAWLS, John. A Theory of Justice, (Revised Edition). Cambridge: Harvard University Press, 1999.

------- A Theory of Justice. Cambridge, Massachusetts: Harvard University Press, Belknap Press, 1971.

SHUSTERMAN, Richard. "Putnam and Cavell on the Ethics of Democracy", en Political Theory, Vol. 25, № 2 (Apr., 1997):193-214.

SIEMENS, Herman W. "Agonal Communities of Taste: Law and Community in Nietzsche's Philosophy of Transvaluation”, en Journal of Nietzsche Studies 24 (2002):83-112.

STIEGLER, Barbara. Nietzsche et la biologie. Paris: P. U. F., 2001. 
Vanessa Lemm

TAYLOR, Quentin P. The Republic of Genius. A Reconstruction of Nietzsche's Early Thought. Rochester: Rochester University Press, 1997.

TOCQUEVILlE, Alexis de. Democracy in America and Two Essays on America. London: Penguin Books, 2003. 